\title{
Heme Effects in Lowering Patient Discomfort in Radial Artery Verapamil Injection
}

\author{
James Livesay ${ }^{\mathrm{a}}$, Raj Baljepally a, b, c, Hassan Tahir ${ }^{\mathrm{a}}$, Robert Heidel ${ }^{\mathrm{a}}$
}

\begin{abstract}
Background: The primary objective of this study was to evaluate whether diluting verapamil with heme as compared to normal saline reduces patient discomfort during radial artery injection. Following radial artery access, verapamil is frequently administered to reduce the incidence of radial artery spasm. The injection of verapamil via the radial artery is associated with a temporary "burning" discomfort. It has been hypothesized that the dilution of verapamil with heme rather than saline leads to less patient discomfort during administration.
\end{abstract}

Methods: This prospective, single-center, randomized study enrolled patients undergoing coronary angiography via radial artery access between August 2017 and April 2018. Patients were randomized 1:1 to receive either verapamil/heme or verapamil/saline administration. Patient discomfort was assessed at the time of injection, and 1-h post procedure.

Results: A total of 214 patients were enrolled in the study. Of these, 103 patients were included in the verapamil/heme group and 100 in the verapamil/saline group. For the primary objectives, there were no statistically significant differences in patient discomfort at the time of verapamil injection ( $\mathrm{P}=0.15)$, or 1 -h post injection $(\mathrm{P}=0.48)$. However, in the verapamil/heme group, there was a trend towards a lower post-injection pain score $(\mathrm{M}=2.83$, standard deviation $(\mathrm{SD})=$ $3.18)$ compared to the verapamil/saline group $(\mathrm{M}=3.48, \mathrm{SD}=3.01)$ $(\mathrm{P}=0.15)$.

Conclusion: There was no significant difference in immediate or 1-h post-procedure patient discomfort with verapamil diluted with heme as compared to dilution with saline. While there was a trend towards lower immediate patient discomfort in the verapamil/heme group, larger studies are needed to further evaluate this trend.

Manuscript submitted July 30, 2021, accepted August 13, 2021

Published online September 9, 2021

aDepartment of Medicine, University of Tennessee Graduate School of Medicine, Knoxville, TN, USA

${ }^{b}$ Heart Lung Vascular Institute, University of Tennessee Medical Center, Knoxville, TN, USA

${ }^{\mathrm{c} C o r r e s p o n d i n g ~ A u t h o r: ~ R a j ~ B a l j e p a l l y, ~ D i v i s i o n ~ o f ~ C a r d i o l o g y, ~ H e a r t ~ L u n g ~}$ Vascular Institute, University of Tennessee Medical Center, 1940 Alcoa Hwy, Suite E-310, Knoxville, TN 37920, USA. Email: RBaljepa@utmck.edu

doi: https://doi.org/10.14740/cr1313
Keywords: Radial access; Verapamil; Radial artery spasm; Radial discomfort; Radial cocktail

\section{Introduction}

The incidence of transradial access (TRA) for coronary angiography continues to increase in the USA [1]. There are now several prospective trials and meta-analysis supporting the use of TRA in acute coronary syndrome (ACS) patients, and particularly in ST-segment myocardial infarction (STEMI) patients [2-4]. In 2015, the European Society of Cardiology updated guidelines regarding the management of patients with ACS, and ultimately recommended TRA (class $1, \mathrm{~A}$ ) as the preferred method of access [5]. With increasing use of TRA as the primary access route for coronary angiography, also comes an increased focus on best practices associated with obtaining and utilizing TRA. One important aspect of utilizing TRA is the complication of radial artery spasm (RAS), which has a reported incidence of 7.5-20\% [6-8]. To prevent RAS, a nondihydropyridine calcium channel blocker (CCB) known as verapamil is commonly injected or included in a "radial cocktail". To date, this use of intra-arterial CCB has demonstrated a reduction in the incidence of RAS [8-11].

Although effective in reducing RAS, the arterial administration of verapamil has been associated with a "burning sensation" and patient discomfort on injection. Furthermore, there is a hypothesis that diluting verapamil with the patient's own blood (heme) results in less patient discomfort when compared to dilution with saline. While the practice of diluting verapamil with heme is anecdotally common, to the best of our knowledge, the effects of verapamil dilution with heme have not previously been studied.

The aim of this randomized, single-center, prospective study was to compare the effects of verapamil dilution with heme versus saline on patient discomfort during radial artery injection.

\section{Materials and Methods}

\section{Study design}

This was a prospective, single-center, randomized, single- 
blinded study designed to compare the comfort-related effects of radial artery injection of heme diluted verapamil versus saline diluted verapamil. The $\mathrm{pH}$ levels at the time of injection were not obtained during this study. A total of 214 patients were enrolled in the study between August 2017 and April 2018. Patients were randomized in a 1:1 single-blinded fashion to either the verapamil/heme or verapamil/saline group. Patient discomfort was assessed both immediately after verapamil injection and 1-h post procedure. The presence and severity of access site arm discomfort was assessed by asking patients to rate ipsilateral arm discomfort using a 0-10 pain scale with 0 meaning the patient experiencing no pain and 10 severe pain. The study was approved by the Institutional Review Board at the University of Tennessee Graduate School of Medicine, Knoxville, TN. The IRB number associated with this study is 4287. This study was conducted in compliance with the ethical standards if the responsible institution on human subjects as well with the Helsinki Declaration.

\section{Patient selection}

All patients over the age of 18 years old presenting for coronary angiography via radial artery access were eligible for enrollment. Exclusion criteria included patients under the age of 18 years old, inability to obtain radial artery access, conversion from radial to alternate access, and patients with planned concomitant venous access. Patients who were unable to clearly answer the pain assessment questions, due to sedation, were also excluded from the final study analysis.

\section{Endpoints}

The primary endpoints of this study were patient discomfort immediately after verapamil injection and $1-\mathrm{h}$ post procedure, as assessed on a standard 0-10 numeric pain scale. Secondary endpoints included the total amount of sedation required, incidence of vasospasm, and the total procedure time.

\section{Statistical analysis}

Percentages and mean with standard deviation (SD) were used to describe the distribution of categorical and continuous variables with normal distribution, respectively. Mean with interquartile range (IQR) were used to describe the distribution of continuous variables that were not normally distributed. Continuous variables were compared between the patients receiving heme diluted verapamil and saline diluted verapamil using two-independent samples $t$-tests or by Mann-Whitney $\mathrm{U}$ test, depending upon the meeting of statistical assumptions (normality and homogeneity of variance). Comparison of categorical variables was performed using the Pearson Chisquared test. Between-subjects comparisons were performed on the following baseline patient characteristics: age, sex, race, presence of coronary artery disease, peripheral vascular disease, chronic kidney disease, diabetes mellitus, hyperten- sion, hyperlipidemia, tobacco use, and baseline medication use. Procedural variables including pre-procedural and total sedation, ultrasound use, number of sticks required for access, amount of lidocaine, amount of nitroglycerine use, and amount of verapamil used were also analyzed using between-subjects comparisons. Post hoc power analyses were performed in the instance that the primary outcomes did not achieve statistical significance. Statistical analyses were performed using SPSS Version 26 (Armonk, NY: IBM Corp.) All P-values are twosided, with $\mathrm{P}<0.05$ indicating statistically significant.

\section{Results}

During the study enrollment period, a total of 214 patients were enrolled in the present study between August 2017 and April 2018. Patients meeting inclusion criteria were randomized 1:1 to either the verapamil/heme or verapamil/saline group. Two hundred three patients, including 103 patients in the verapamil/heme group and 100 patients in the verapamil/ saline group, were included in the final statistical analysis after exclusion criteria were applied. The primary outcome of the study was patient discomfort, assessed by numerical pain score rating $(0-10)$ at the time of verapamil injection as well as $1-\mathrm{h}$ post procedure.

The demographic and patient clinical characteristics between the verapamil/heme or verapamil/saline groups were well matched (Table 1) with mean age 65.05 vs. $64.68(\mathrm{P}=$ $0.80)$, and $59.0 \%$ vs. $61.2 \%$ were male $(\mathrm{P}=0.75)$. The majority of patients included in the study were non-ACS patients, with $15.3 \%(n=31)$ of patients meeting criteria for ACS. Of the patients with ACS, there were more ACS patients in the verapamil/heme group $(\mathrm{n}=22,21.4 \%)$, compared to the verapamil/saline group $(n=9,9.0 \%)$. Both groups had similar use of medications, except CCB $(13 \%$ in verapamil/saline group vs. $25 \%$ in the verapamil/heme group, $\mathrm{P}=0.027)(\mathrm{Ta}-$ ble 2).

In terms of procedural characteristics (Tables 3 and 4), there was a low incidence of symptomatic RAS of $1.0 \%$, with only one patient experiencing RAS in each group. There was higher total versed dose in the heme group compared to the saline group but not statistically significant $(3.0 \mathrm{vs} .2 .0 \mathrm{mg}$, $\mathrm{P}=0.08)$. There was a higher mean dose of verapamil in the heme group compared to the saline group (2.61 vs. 2.34 $\mathrm{mg}, \mathrm{P}=0.02$ ) which was statistically significant (Fig. 1 ). The overall procedure time was slightly higher in the heme/verapamil group, but this was not statistically significant $(\mathrm{P}=$ 0.53 ) (Fig. 2). There were no statistically significant differences for the remainder of the procedural variables between the two groups.

The primary endpoints of the study included patient discomfort immediately post verapamil injection, and 1-h post procedure. There was no statistically significant difference between the two groups in the immediate pain score assessment $(\mathrm{P}=0.15$, post hoc power $=0.318)($ Fig. 3$)$ or 1 -h post procedure $(\mathrm{P}=0.48$, post hoc power $=0.148)($ Fig. 4 , Table 4$)$. In the immediate pain score assessment, there was a lower overall pain score rating in the verapamil/heme group compared to the 
Table 1. Baseline Characteristics of Patients

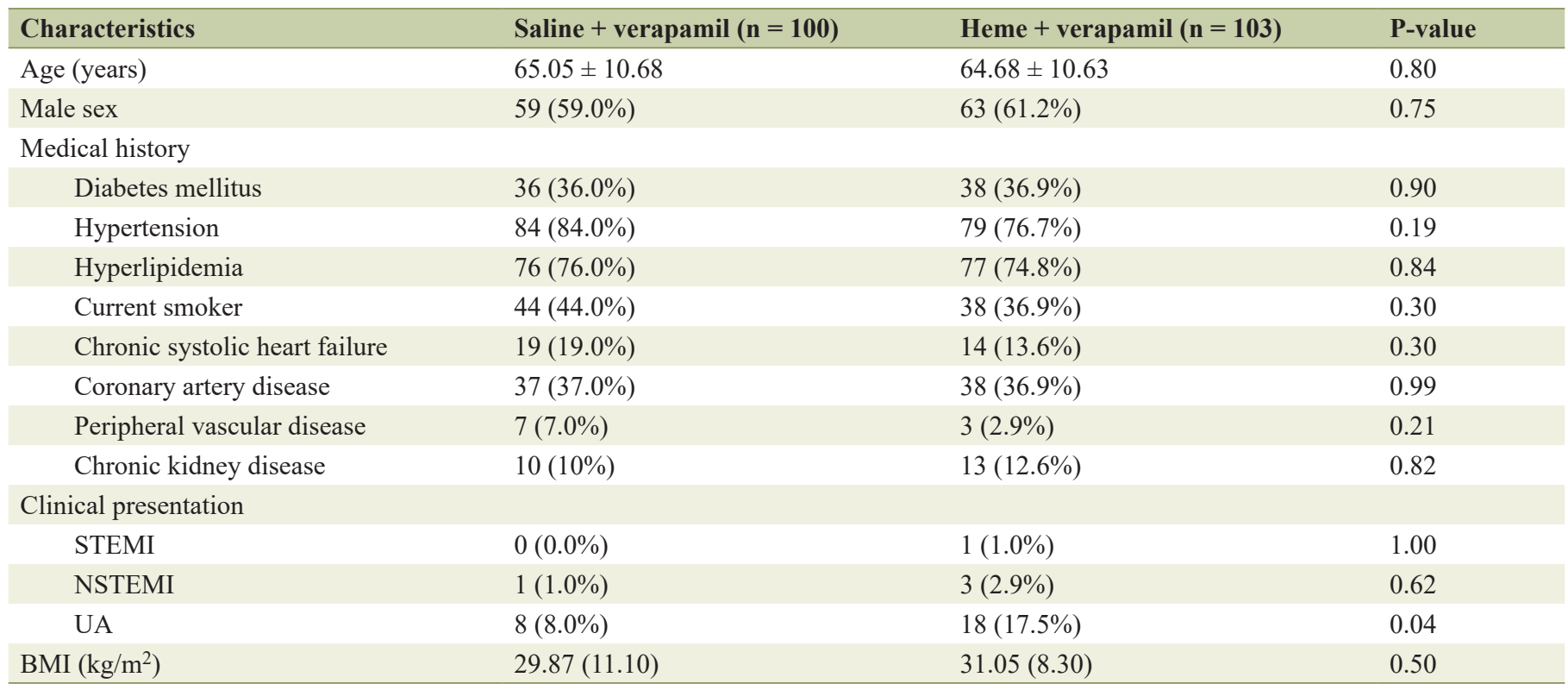

Variables are expressed as no. (\%) or mean \pm standard deviation. $\mathrm{P}<0.05$ indicates statistically significant difference between the groups. STEMI: ST elevation myocardial infarction; NSTEMI: non-ST elevation myocardial infarction; UA: unstable angina; BMI: body mass index.

Table 2. Chronic Medications by Patient Groups

\begin{tabular}{llll}
\hline Medications & Saline + verapamil $(\mathbf{n}=\mathbf{1 0 0})$ & Heme + verapamil $(\mathbf{n}=\mathbf{1 0 3})$ & P-value \\
\hline Aspirin & $63(63.0 \%)$ & $57(55.3 \%)$ & 0.27 \\
Clopidogrel & $9(9.0 \%)$ & $10(9.7 \%)$ & 0.86 \\
Prasugrel & $2(2.0 \%)$ & $5(4.9 \%)$ & 0.45 \\
Ticagrelor & $2(2.0 \%)$ & $3(2.9 \%)$ & 1.00 \\
Warfarin & $10(10.0 \%)$ & $7(6.8 \%)$ & 0.41 \\
Apixaban & $5(5.0 \%)$ & $7(6.8 \%)$ & 0.59 \\
Rivaroxaban & $2(2.0 \%)$ & $4(3.9 \%)$ & 0.68 \\
Endoxaban & $1(1.0 \%)$ & $0(0.0 \%)$ & 0.49 \\
Beta-blocker & $61(61.0 \%)$ & $64(62.1 \%)$ & 0.87 \\
CCB & $13(13.0 \%)$ & $26(25.2 \%)$ & 0.027 \\
ACEI/ARB & $51(51.0 \%)$ & $49(47.6 \%)$ & 0.63 \\
Statin & $63(63.0 \%)$ & $65(63.1 \%)$ & 0.99 \\
\hline
\end{tabular}

Variables are expressed as no. (\%). P < 0.05 indicates statistically significant difference between the groups. CCB: calcium channel blocker; ACEI: angiotensin-converting-enzyme inhibitors; ARB: angiotensin II receptor blockers.

Table 3. Procedural Data by Patient Groups

\begin{tabular}{llll} 
Variables & Saline + verapamil $(\mathbf{n}=\mathbf{1 0 0})$ & Heme + verapamil $(\mathbf{n}=\mathbf{1 0 3})$ & P-value \\
\hline Ultrasound used & $12(12.0 \%)$ & $11(10.7 \%)$ & 0.77 \\
PCI & $12(12.0 \%)$ & $18(17.5 \%)$ & 0.27 \\
Nitro $(\mu \mathrm{g})$ & $230.0 \pm 87.5$ & $217 \pm 100$ & 0.59 \\
Verapamil $(\mathrm{mL})$ & $2.34 \pm 0.83$ & $2.61 \pm 0.79$ & 0.02 \\
Lidocaine $(\mathrm{mL})$ & $1.4 \pm 1.6$ & $1.3 \pm 1.43$ & 0.58 \\
Number of sticks & $1.54 \pm 1.007$ & $1.71 \pm 1.0$ & 0.77 \\
\hline
\end{tabular}

Variables are expressed as no. (\%) or mean \pm standard deviation. $\mathrm{P}<0.05$ indicates statistically significant difference between the groups. $\mathrm{PCl}$ : percutaneous coronary intervention. 
Table 4. Comparison of Independent Groups on Primary and Secondary Endpoints

\begin{tabular}{llll}
\hline Outcomes & Saline + verapamil $(\mathbf{n}=\mathbf{1 0 0})$ & Heme + verapamil $(\mathbf{n}=\mathbf{1 0 3})$ & P-value \\
\hline Injection pain score & $3.48 \pm 3.01$ & $2.83 \pm 3.18$ & 0.15 \\
1-h pain score & $0.80 \pm 1.78$ & $1.0 \pm 2.31$ & 0.48 \\
Pre-versed $(\mathrm{mg})$ & $1.5 \pm 1.2$ & $2.4 \pm 2.1$ & 0.016 \\
Pre-fentanyl $(\mu \mathrm{g})$ & $50.65 \pm 50.0$ & $53.23 \pm 52.12$ & 0.60 \\
Pre-benadryl $(\mathrm{mg})$ & $33.25 \pm 23.58$ & $29.85 \pm 24.52$ & 0.32 \\
Total versed $(\mathrm{mg})$ & $2.75 \pm 2.38$ & $3.0 \pm 2.0$ & 0.08 \\
Total fentanyl $(\mu \mathrm{g})$ & $89.67 \pm 55.31$ & $93.45 \pm 55.39$ & 0.61 \\
Total benadryl $(\mathrm{mg})$ & $33.81 \pm 23.51$ & $29 \pm 24.56$ & 0.15 \\
Total procedure time $(\mathrm{min})$ & $21.5 \pm 21.0$ & $24.0 \pm 20.0$ & 0.53 \\
Vasospasm & $3(3.0 \%)$ & $0(0.0 \%)$ & 0.12 \\
Hematoma & $1(1.0 \%)$ & $1(1.0 \%)$ & 1.00 \\
\hline
\end{tabular}

Variables are expressed as no. (\%) or mean \pm standard deviation. $\mathrm{P}<0.05$ indicates statistically significant difference between the groups.

verapamil/saline group $(\mathrm{M}=2.83, \mathrm{SD}=3.18$ vs. $\mathrm{M}=3.48, \mathrm{SD}$ $=3.01$, respectively) (Fig. 2).

\section{Discussion}

In this prospective, randomized, single-center study, we evaluated the effects of diluting verapamil with heme compared to saline on patient discomfort during radial artery injection. As stated, increasing use of TRA in the USA has also increased focus on reducing access and catheterization associated radial artery complications. RAS is one of the major concerns related to radial artery use and is reduced with intraarterial verapamil administration [8, 9, 12-14]. An association between verapamil administration and arm discomfort has been anecdotally noted in the cardiovascular community;

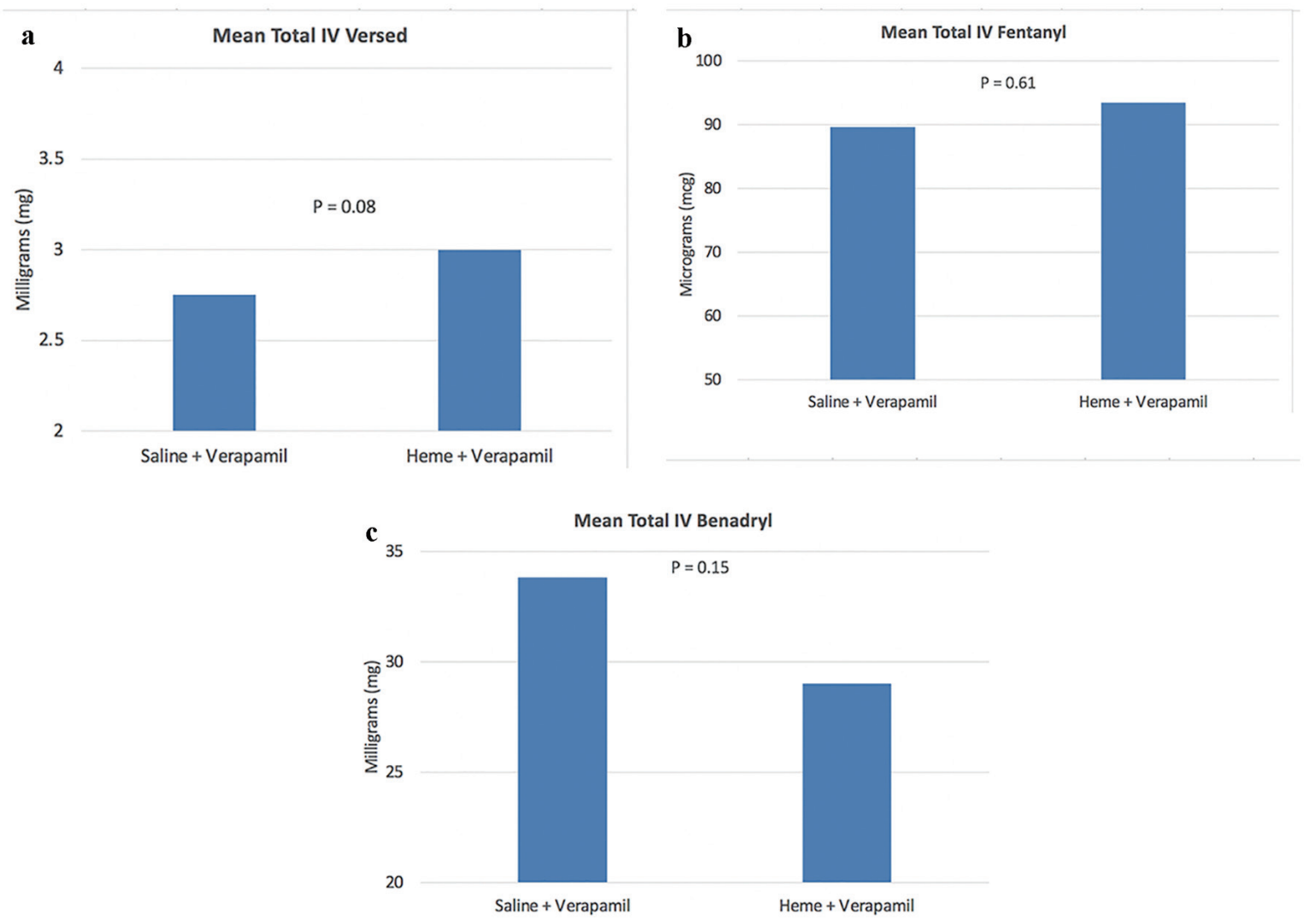

Figure 1. Graphs of total sedation between the two groups. 


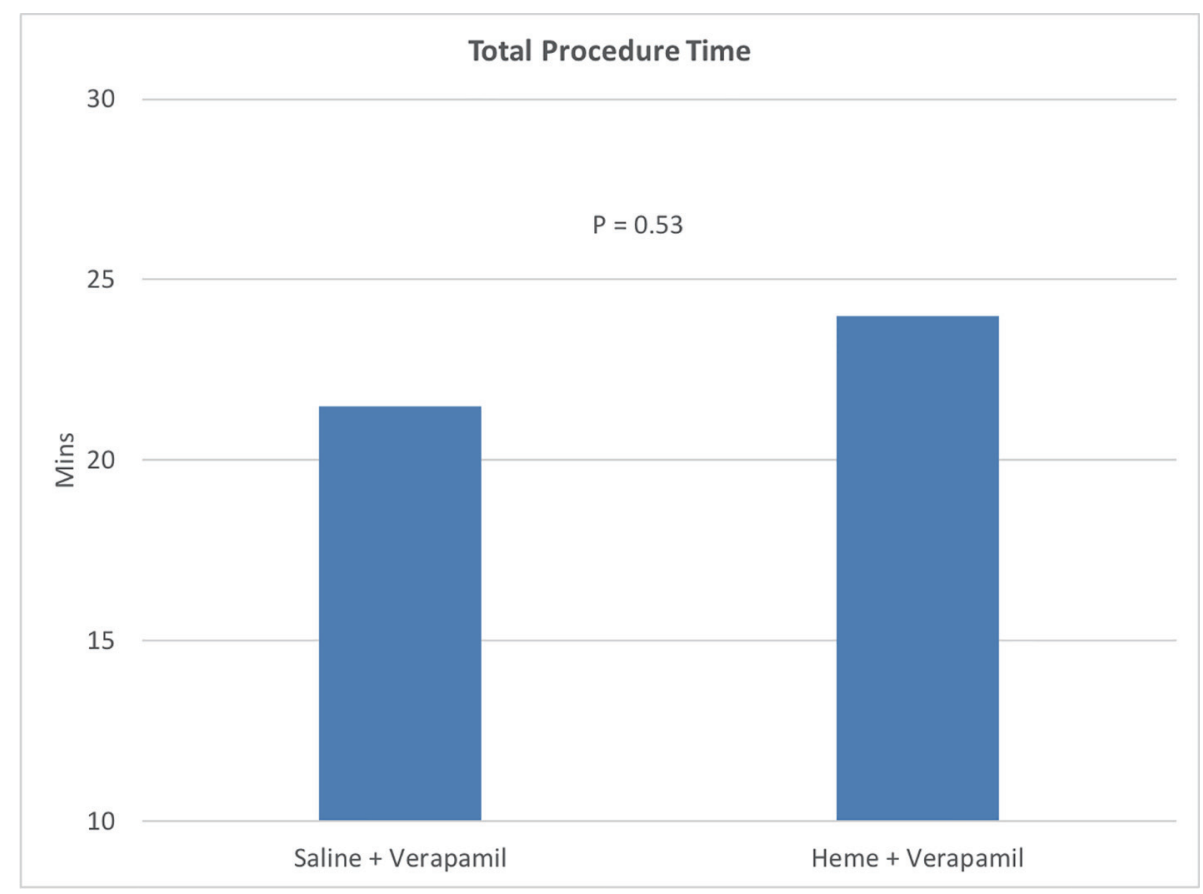

Figure 2. Graph of total procedure time between the two groups.

$$
P=0.15
$$

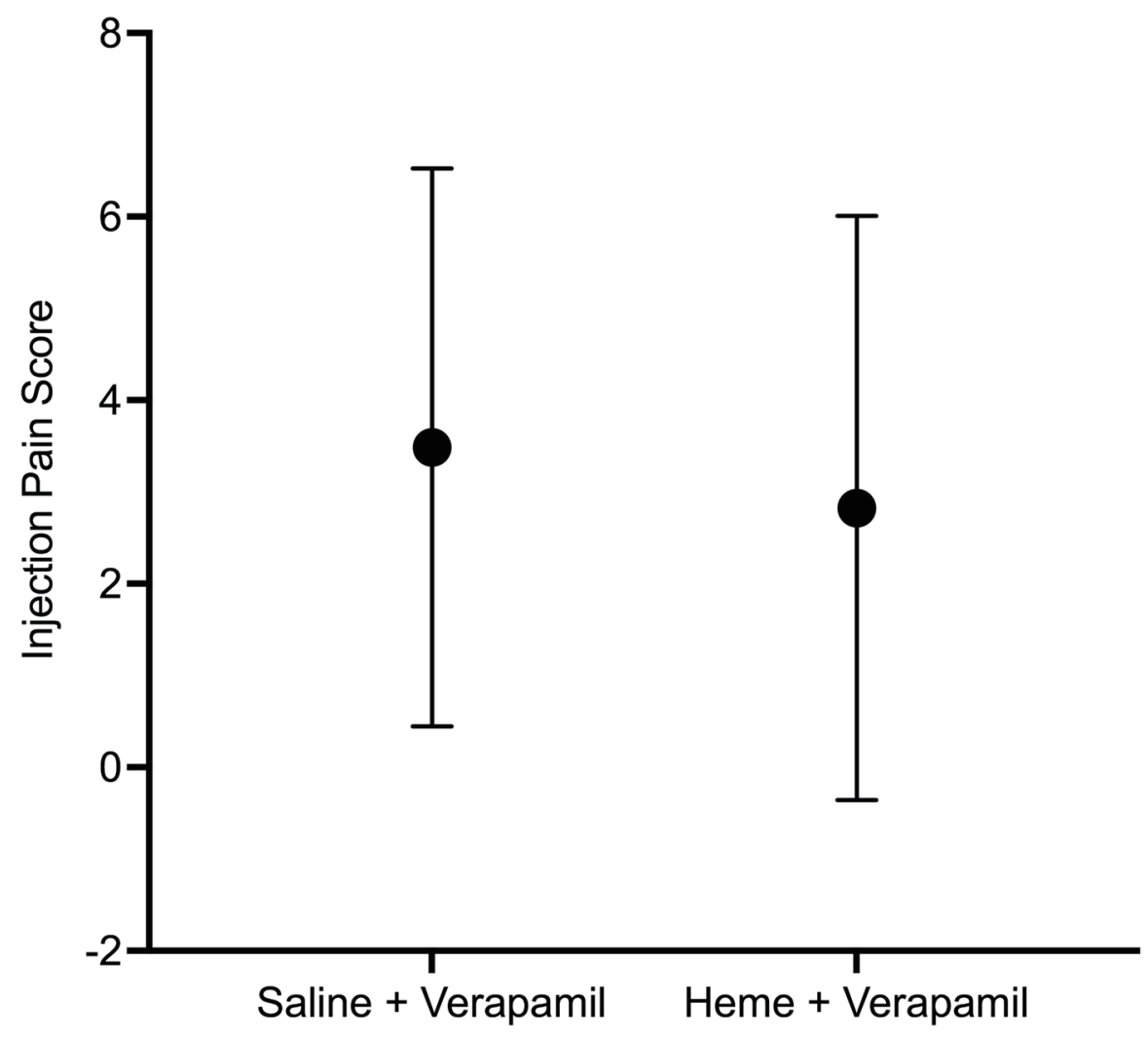

Figure 3. Injection pain score between the two groups. 


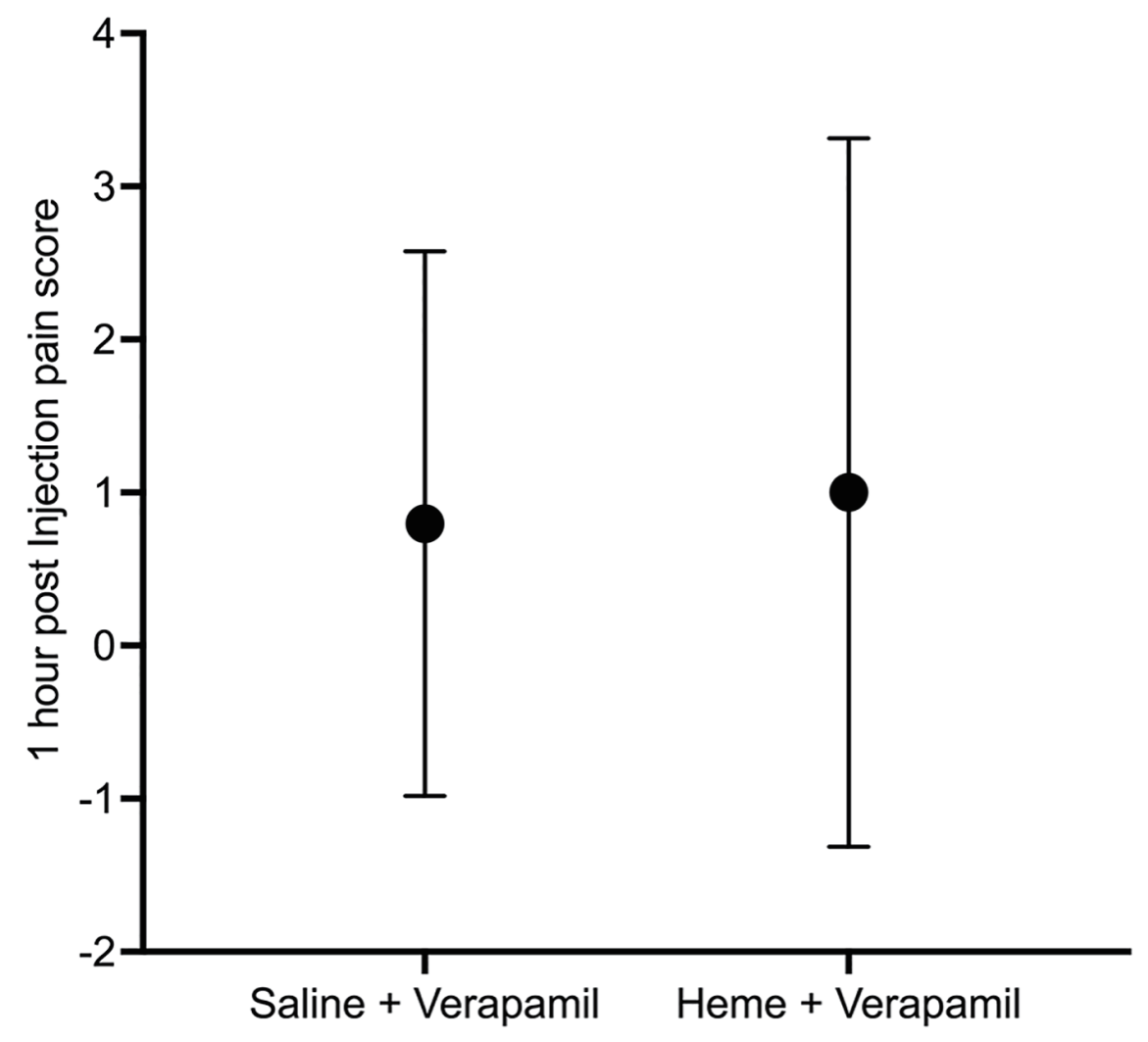

Figure 4. One-hour pain score between the two groups.

however, to the best of our knowledge, there are no randomized studies evaluating this association or possible remedies to it.

This study explored such associations. We did not observe any statistically significant differences, in patient discomfort, between the verapamil/heme and verapamil/saline groups either immediately post injection or 1-h post procedure. We did, however, observe a trend towards lower average pain scores immediately post injection in the verapamil/ heme group compared to the verapamil/saline group. The lack of statistical significance may potentially be due to the low sample size of this study. Two confounding variables identified in this study are a higher average dose of both versed and verapamil used in the heme group, which may have affected the primary outcome of the study, although notably the differences were well below known clinically effective dose escalation protocols.

\section{Conclusion}

With increasing use of TRA, there is an increasing focus on improving best practices and reducing associated complications while maximizing patient satisfaction. Verapamil diluted with heme is a common practice among invasive cardiologists to lower discomfort in the ipsilateral arm. Our study did not find any significant difference in patient discomfort with injection of intra-arterial verapamil/heme compared to verapamil/ saline via the radial artery. Our data did suggest a trend towards lower immediate post-injection patient discomfort when diluting verapamil with heme; however, larger studies are required to further evaluate whether this is truly a statistically significant effect on patient discomfort.

\section{Acknowledgments}

Bryon Cook, MD and Rajiv Sharma, MD helped with obtaining patient consent.

\section{Financial Disclosures}

None to declare.

\section{Conflict of Interest}

The authors report no conflict of interest regarding the content herein. 


\section{Informed Consent}

All participants of the study signed an informed consent prior to inclusion in the study.

\section{Author Contributions}

James Livesay and Raj Baljepally conceived and designed the study, assisted with patient consents, and wrote the manuscript. Hassan Tahir and Robert Heidel performed the statistical analysis and helped in writing the methods and results section of the manuscript.

\section{Data Availability}

The data supporting these findings of this study are available from the corresponding author upon reasonable request.

\section{References}

1. Masoudi FA, Ponirakis A, de Lemos JA, Jollis JG, Kremers M, Messenger JC, Moore JWM, et al. Trends in U.S. cardiovascular care: 2016 report from 4 ACC national cardiovascular data registries. J Am Coll Cardiol. 2017;69(11):1427-1450.

2. Jolly SS, Yusuf S, Cairns J, Niemela K, Xavier D, Widimsky P, Budaj A, et al. Radial versus femoral access for coronary angiography and intervention in patients with acute coronary syndromes (RIVAL): a randomised, parallel group, multicentre trial. Lancet. 2011;377(9775):1409-1420.

3. Bernat I, Horak D, Stasek J, Mates M, Pesek J, Ostadal P, Hrabos V, et al. ST-segment elevation myocardial infarction treated by radial or femoral approach in a multicenter randomized clinical trial: the STEMI-RADIAL trial. J Am Coll Cardiol. 2014;63(10):964-972.

4. Ferrante G, Rao SV, Juni P, Da Costa BR, Reimers B, Condorelli G, Anzuini A, et al. Radial versus femoral access for coronary interventions across the entire spectrum of patients with coronary artery disease: a metaanalysis of randomized trials. JACC Cardiovasc Interv. 2016;9(14):1419-1434.

5. Roffi M, Patrono C, Collet JP, Mueller C, Valgimigli M,
Andreotti F, Bax JJ, et al. 2015 ESC Guidelines for the management of acute coronary syndromes in patients presenting without persistent ST-segment elevation: task force for the management of acute coronary syndromes in patients presenting without persistent ST-segment elevation of the European Society of Cardiology (ESC). Eur Heart J. 2016;37(3):267-315.

6. Jia DA, Zhou YJ, Shi DM, Liu YY, Wang JL, Liu XL, Wang ZJ, et al. Incidence and predictors of radial artery spasm during transradial coronary angiography and intervention. Chin Med J (Engl). 2010;123(7):843-847.

7. Ruiz-Salmeron RJ, Mora R, Velez-Gimon M, Ortiz J, Fernandez C, Vidal B, Masotti M, et al. [Radial artery spasm in transradial cardiac catheterization. Assessment of factors related to its occurrence, and of its consequences during follow-up]. Rev Esp Cardiol. 2005;58(5):504-511.

8. Kwok CS, Rashid M, Fraser D, Nolan J, Mamas M. Intra-arterial vasodilators to prevent radial artery spasm: a systematic review and pooled analysis of clinical studies. Cardiovasc Revasc Med. 2015;16(8):484-490.

9. Curtis E, Fernandez R, Lee A. The effect of vasodilatory medications on radial artery spasm in patients undergoing transradial coronary artery procedures: a systematic review. JBI Database System Rev Implement Rep. 2017;15(7):1952-1967.

10. Gorgulu S, Norgaz T, Karaahmet T, Dagdelen S. Incidence and predictors of radial artery spasm at the beginning of a transradial coronary procedure. J Interv Cardiol. 2013;26(2):208-213.

11. Boyer N, Beyer A, Gupta V, Dehghani H, Hindnavis V, Shunk K, Zimmet J, et al. The effects of intra-arterial vasodilators on radial artery size and spasm: implications for contemporary use of trans-radial access for coronary angiography and percutaneous coronary intervention. Cardiovasc Revasc Med. 2013;14(6):321-324.

12. Aminian A, Lalmand J, Dolatabadi D. Prevention of radial artery spasm: importance of a multifactorial approach. JACC Cardiovasc Interv. 2013;6(11):1214.

13. Chen CW, Lin CL, Lin TK, Lin CD. A simple and effective regimen for prevention of radial artery spasm during coronary catheterization. Cardiology. 2006;105(1):43-47.

14. Varenne O, Jegou A, Cohen R, Empana JP, Salengro E, Ohanessian A, Gaultier C, et al. Prevention of arterial spasm during percutaneous coronary interventions through radial artery: the SPASM study. Catheter Cardiovasc Interv. 2006;68(2):231-235. 